\title{
RIGIDITY RESULTS \\ FOR ELLIPTIC BOUNDARY VALUE PROBLEMS: STABLE SOLUTIONS FOR QUASILINEAR EQUATIONS WITH NEUMANN OR ROBIN BOUNDARY CONDITIONS
}

\author{
SERENA DIPIERRO, ANDREA PINAMONTI, ENRICO VALDINOCI
}

\begin{abstract}
We provide a general approach to the classification results of stable solutions of (possibly nonlinear) elliptic problems with Robin conditions.

The method is based on a geometric formula of Poincaré type, which is inspired by a classical work of Sternberg and Zumbrun and which gives an accurate description of the curvatures of the level sets of the stable solutions. From this, we show that the stable solutions of a quasilinear problem with Neumann data are necessarily constant.

As a byproduct of this, we obtain an alternative proof of a celebrated result of Casten and Holland, and Matano.

In addition, we will obtain as a consequence a new proof of a result recently established by Bandle, Mastrolia, Monticelli and Punzo.
\end{abstract}

\section{Contents}

1. Introduction

2. A geometric Poincaré inequality: proofs of Theorem 1.3 and Corollary 1.4

3. Proof of Theorem 1.5

4. Proof of Theorem 1.6

References

\section{INTRODUCTION}

The study of stable solutions of variational problems is a classical topic of investigation. Roughly speaking, stable solutions are critical points of an energy functional with positive second variation (in particular, local minimizers of the energy are stable solutions).

Given their special energetic properties, stable solutions often enjoy better qualitative and quantitative properties than the other solutions and, in some cases, they can be completely classified. We refer to the monograph [13] and the references therein for a complete presentation of the main results available on stable solutions.

Part of this work was done while A. P. was visiting the Dipartimento di Matematica "Federigo Enriques" of the Università di Milano. The authors are members of Gruppo Nazionale per l'Analisi Matematica, la Probabilità e le loro Applicazioni (GNAMPA) of the Istituto Nazionale di Alta Matematica (INdAM). The first and third authors are supported by the Australian Research Council grant "N.E.W." Nonlocal Equation at Work. 
The goal of this paper is to provide an approach towards the classification of stable solutions with Neumann and Robin boundary conditions which is based on a geometric Poincaré formula. This formula is inspired by the one originally introduced by Sternberg and Zumbrun in 40, 41 and provides an accurate bound on the second fundamental form and on the tangential derivatives of the level sets of any stable solution, in a form which is remarkably independent from the nonlinearity. For this reason, we think that the geometric formula obtained is interesting in itself and reveals important information on the level sets of the solutions.

With this approach, we obtain a classification result for (possibly nonlinear) elliptic problems with Neumann boundary conditions (these operators can be also singular or degenerate, and we comprise the cases of the $p$-Laplacian and of the mean curvature equation). This result contains, as a particular case, the classical one obtained by Casten and Holland in [4] and by Matano in [35], for which we produce a different proof (in fact, with weaker regularity assumptions).

In addition, the method also gives a classification result that has been recently obtained by Bandle, Mastrolia, Monticelli and Punzo in [2, see also [11].

The mathematical framework in which we work is the following. Let $\Omega \subset \mathbb{R}^{n}$ be a bounded domain with smooth boundary. We study the solutions to the following boundary value problem:

$$
\left\{\begin{aligned}
\operatorname{div}(a(|\nabla u|) \nabla u)+f(u)=0 & \text { in } \Omega \\
a(|\nabla u|) \partial_{\nu} u+h(u)=0 & \text { on } \partial \Omega
\end{aligned}\right.
$$

where $f, h \in C^{1}(\mathbb{R})$ and $a \in C_{l o c}^{1,1}((0,+\infty))$. Here $\nu$ denotes the external unit normal to $\partial \Omega$. We also require that the function $a$ satisfies the following structural conditions:

$$
\begin{aligned}
& a(t)>0 \text { for any } t \in(0,+\infty), \\
& a(t)+a^{\prime}(t) t>0 \text { for any } t \in(0,+\infty) .
\end{aligned}
$$

We observe that the general form of (11) and the structural conditions (21) and (3) encompass, as very special cases, many elliptic singular and degenerate equations. Indeed, if $a(t):=t^{p-2}$, with $1<p<+\infty$, or $a(t):=1 / \sqrt{1+t^{2}}$, then we obtain the $p$-Laplacian and the mean curvature equations respectively.

Following [14, 17, 5], we define $A: \mathbb{R}^{n} \rightarrow \operatorname{Mat}(n \times n)$ and $\lambda_{1} \in C^{0}((0,+\infty))$ as follows

$$
\begin{gathered}
A_{h k}(\xi):=\frac{a^{\prime}(|\xi|)}{|\xi|} \xi_{h} \xi_{k}+a(|\xi|) \delta_{h k} \quad \text { for any } 1 \leq h, k \leq n, \\
\lambda_{1}(t):=a(t)+a^{\prime}(t) t \quad \text { for any } t>0 .
\end{gathered}
$$

Definition 1.1. We say that $u$ is a weak solution to (11) if $u \in C^{1}(\bar{\Omega})$,

$$
\int_{\Omega} a(|\nabla u|)\langle\nabla u, \nabla \varphi\rangle d x+\int_{\partial \Omega} h(u) \varphi d \sigma-\int_{\Omega} f(u) \varphi d x=0,
$$

for any $\varphi \in C^{1}(\bar{\Omega})$, and either (A1) or (A2) is satisfied, where

(A1) $\{\nabla u=0\}=\varnothing$; 
(A2) $a \in C^{0}([0,+\infty))$ and

$$
\text { the map } t \rightarrow t a(t) \text { belongs to } C^{1}([0,+\infty)) .
$$

Notice that the first integrand in ([6) is well-defined, thanks to either (A1) or (A2) (recall the boundary condition in (10).

We observe that, in general, equation (11) may have no solution. For instance, in the mean curvature equation case in which $a(t):=\frac{1}{\sqrt{1+t^{2}}}$, a compatibility condition was discovered in [26], see also Theorem 1.3 and Remark 1.4 in [34]: in particular, if $f$ is a nonzero constant and $h:=0$, there is no solution.

The regularity assumption $u \in C^{1}(\bar{\Omega})$ is always fulfilled in many important cases, like those involving the $p$-Laplacian operator or the mean curvature operator (see e.g. [33]).

In light of this, and in view of the great generality of the functions $a$ and $h$, it is natural to work in the above setting.

Definition 1.2. Let $u$ be a weak solution to (11). We say that $u$ is stable if

$$
\int_{\Omega}\langle A(\nabla u) \nabla \varphi, \nabla \varphi\rangle d x+\int_{\partial \Omega} h^{\prime}(u) \varphi^{2} d \sigma-\int_{\Omega} f^{\prime}(u) \varphi^{2} d x \geq 0
$$

for any $\varphi \in C^{1}(\bar{\Omega})$.

We notice that, as customary, from the variational point of view, such definition of stability is equivalent to the fact that the associated energy functional is nonnegative defined (nevertheless, we do not need to explicitly introduce such energy setting, since the framework provided by equation (11), combined with the condition in (7), is sufficient for our purposes).

In the subsequent formula (9), we give an extension of the geometric formula obtained in [40, 41]. Such formula relates the stability of the equation with the principal curvatures of the corresponding level set and with the tangential gradient of the solution. Since this formula bounds a weighted $L^{2}$-norm of any $\varphi \in C^{1}(\bar{\Omega})$ plus a boundary term by a weighted $L^{2}$-norm of its gradient, we may see it as a weighted Poincaré type inequality.

The first result towards a geometric Poincaré inequality is the following:

Theorem 1.3. Let $u \in C^{2}(\bar{\Omega})$ be a stable weak solution to (1). Then, for every $\varphi \in C^{1}(\bar{\Omega})$, it holds

$$
\begin{aligned}
& \int_{\{\nabla u \neq 0\} \cap \Omega}\left[\sum_{i=1}^{n}\left\langle A(\nabla u) \nabla u_{i}, \nabla u_{i}\right\rangle-\langle A(\nabla u) \nabla|\nabla u|, \nabla|\nabla u|\rangle\right] \varphi^{2} d x \\
& \quad+\int_{\partial \Omega}\left(f(u) \partial_{\nu} u-a(|\nabla u|)\left\langle\nabla u, \partial_{\nu} \nabla u\right\rangle-h(u) \Delta u-h^{\prime}(u)|\nabla u|^{2}\right) \varphi^{2} d \sigma \\
& \leq \int_{\Omega}|\nabla u|^{2}\langle A(\nabla u) \nabla \varphi, \nabla \varphi\rangle d x .
\end{aligned}
$$

Concerning the regularity assumption on the solution taken in Theorem 1.3, we think that it is an interesting problem to determine whether similar results can be obtained under weaker regularity. This would be particularly interesting in the case of $p$-Laplacetype equations, in which the gradient of the solution is usually not better than Hölder 
continuous at critical points. On the one hand, in our arguments, assuming the continuity of the second derivatives makes the computations available "up to the boundary" and gives perfect sense of the terms $a(|\nabla u|)\left\langle\nabla u, \partial_{\nu} \nabla u\right\rangle$ and $h(u) \Delta u$ along $\partial \Omega$. On the other hand, it is possible that a more careful analysis provides a suitable meaning for these terms also in a less regular situation: for instance, at points where $h(u) \neq 0$, the Robin boundary condition suggests that $\nabla u \neq 0$, which makes the elliptic regularity available (thus reducing to the smooth case), while at points where $h(u)=0$ the term $h(u) \Delta u$ formally disappears. Similarly, at critical points, the term $a(|\nabla u|)\left\langle\nabla u, \partial_{\nu} \nabla u\right\rangle$ may disappear under weaker regularity assumptions than those requested in Theorem 1.3. or have a useful sign at least in the case of convex domains (see Lemma 3.1). These observations indeed suggest that the results presented in this paper may be valid in further generality.

We also observe that the integrand in the first line of (8) has indeed a geometric interpretation in terms of the curvatures of the level sets of $u$ and the tangential gradient.

For this, given a point $x \in \mathbb{R}^{n}$, we let

$$
L_{u, x}:=\left\{y \in \mathbb{R}^{n} \text { s.t. } u(y)=u(x)\right\}
$$

be the level set of $u$ passing through $x$. Furthermore, we denote by $\nabla_{T} u$ the tangential gradient of $u$ along $L_{u, x} \cap\{\nabla u \neq 0\}$, and by $k_{1}, \ldots, k_{n-1}$ the principal curvatures of $L_{u, x} \cap$ $\{\nabla u \neq 0\}$.

With this notation, we have the following:

Corollary 1.4. Let $u \in C^{2}(\bar{\Omega})$ be a stable weak solution to (1). Then, for every $\varphi \in$ $C^{1}(\bar{\Omega})$, it holds

$$
\begin{aligned}
& \int_{\Omega}\left[\left.\lambda_{1}\left|\nabla_{T}\right| \nabla u\right|^{2}+a(|\nabla u|)|\nabla u|^{2} \sum_{j=1}^{n-1} k_{j}^{2}\right] \varphi^{2} d x \\
& \quad+\int_{\partial \Omega}\left(f(u) \partial_{\nu} u-a(|\nabla u|)\left\langle\nabla u, \partial_{\nu} \nabla u\right\rangle-h(u) \Delta u-h^{\prime}(u)|\nabla u|^{2}\right) \varphi^{2} d \sigma \\
& \leq \int_{\Omega}|\nabla u|^{2}\langle A(\nabla u) \nabla \varphi, \nabla \varphi\rangle d x .
\end{aligned}
$$

As already mentioned, this type of formulas has been originally introduced by Sternberg and Zumbrun in [40, 41], and then used to prove symmetry and rigidity results in [14, 17, 18.

Since then, this type of inequalities has been applied in several contexts: to prove rigidity results for boundary reaction-diffusion equations, see [38, 39] (where a celebrated conjecture of De Giorgi for equations driven by the fractional Laplacian is also proved in dimension 2), to handle semilinear equations in Riemannian and Sub-Riemannian spaces, see [15, 19, 20, 24, 25, 37, to study problems involving the Ornstein-Uhlenbeck operator, see [6], as well as semilinear equations with unbounded drift [5, 16] and systems of PDEs [22, 8, 9, 10].

Recently, in [12], the case of Neumann boundary condition for boundary reactiondiffusion equations was dealt with the use of a Poincaré inequality involving also a boundary term. 
Now, we present some rigidity results, in the spirit of [4, by taking advantage of the geometric information given by formula (9).

We first deal with the case of the Neumann boundary condition, that is we choose $h:=0$ in (11). In this setting we get the following result:

Theorem 1.5. Let $\Omega \subset \mathbb{R}^{n}$ be a bounded domain with smooth boundary. Suppose that $\Omega$ is convex, with strictly positive principal curvatures along $\partial \Omega$.

Let $u \in C^{2}(\bar{\Omega})$ be a stable weak solution to (1) with $h:=0$. Then $u$ is constant in $\Omega$.

Theorem 1.5 is new even in the case of the $p$-Laplacian, but our proof is robust enough to deal with a very general class of operators.

Also, we point out that Theorem 1.5 provides, as a byproduct, a new proof of Theorem 1 in [4] (see also [35]) when the function $a:=1$ and so the general operator in (11) boils down to the Laplacian.

As a corollary of Theorem 1.3, we also give an alternative proof of Theorem 2.1 in 2 (see also [27]). More precisely, we deal with the case of the Laplacian in dimension 2 with Robin boundary condition, and we obtain:

Theorem 1.6. Let $\Omega \subset \mathbb{R}^{2}$ be a bounded domain with smooth boundary. Let $u \in C^{2}(\bar{\Omega})$ be a solution to (1) with $a:=1$ and $h(u):=\alpha u$, for some $\alpha \in \mathbb{R}$.

Suppose that

$$
\int_{\partial \Omega} \alpha^{2} u^{2}\left(\frac{f(u)}{\alpha u}-\kappa+\alpha\right) d \sigma<0 \quad \text { and } \quad \alpha+\kappa \geq 0 \text { on } \partial \Omega,
$$

where $\kappa$ is the curvature of $\partial \Omega$.

Then $u$ is unstable.

It would be desirable to grasp a full understanding of the stable solutions of general quasilinear equations and of the role played by the geometry of the domain (with respect to this, it is likely that the convexity assumption can be relaxed, see e.g. the comments in the Remark before formula (13) in [4]). To keep in mind some examples, we observe that:

- The convexity assumption in Theorem 1.5 cannot be completely removed. Indeed, suitable "dumbbell" domains which produce nonconstant stable solutions of (1) have been constructed in Section 6 of [35] (see in particular the figure on page 452 of [35] and Remark 6.4 on page 453 of [35]). The original examples of [35] took into account connected regions with quite complex shapes, and simpler examples have been investigated also in [23, 42, 28, 29, 30, 36, 7, 1, 3].

- If $\mathbb{R} \ni x \mapsto u(x)$ is a smooth function such that $u(x)=-1$ if $x \leq-1, u(x)=1$ if $x \geq 1$ and $0 \leq u^{\prime} \leq 4$, and if $a(t)=0$ if $t \in[0,4]$, then $u$ is a solution of (1) in $\Omega:=(-2,2)$ with $h:=0$ and $f:=0$, which is not constant. This says that the nondegeneracy of the coefficient $a$ cannot be completely removed in the statement of Theorem 1.5.

- The thesis in Theorem 1.6 is quite strong, since it also excludes constant solutions. This is due to condition (10). For instance, the function vanishing identically is a stable solution of (1) with $a:=1, f:=0$ and $h(u):=\alpha u$, for any $\alpha \geq 0$. But this solution does not verify the first condition in (10). 
The paper is organized as follows. In Section 2 we prove Theorem 1.3 and Corollary 1.4 . Then, Sections 3 and 4 contain the proofs of Theorems 1.5 and 1.6 .

\section{A geometric Poincaré inequality: proofs of Theorem 1.3 and Corollary 1.4}

In this section we deal with the Poincaré-type inequality and we give the proof of Theorem 1.3 and Corollary 1.4 .

We start recalling the following result, which has been proved in [17.

Lemma 2.1. For any $\xi \in \mathbb{R}^{n} \backslash\{0\}$, the matrix $A(\xi)$ is symmetric and positive definite, and its eigenvalues are $\lambda_{1}(|\xi|), \cdots, \lambda_{n}(|\xi|)$, where $\lambda_{1}$ is as in (5) and $\lambda_{i}(t):=a(t)$ for every $i=2, \ldots, n$. Moreover,

$$
\langle A(\xi) \xi, \xi\rangle=|\xi|^{2} \lambda_{1}(|\xi|)
$$

and

$$
\langle A(\xi)(V-W),(V-W)\rangle=\langle A(\xi) V, V\rangle+\langle A(\xi) W, W\rangle-2\langle A(\xi) V, W\rangle,
$$

for any $V, W \in \mathbb{R}^{n}$ and any $\xi \in \mathbb{R}^{n} \backslash\{0\}$.

The forthcoming formula (11) is a fundamental step towards the proof of Theorem 1.3 . We let $\nu=\left(\nu_{1}, \ldots, \nu_{n}\right)$ be the unit normal to $\partial \Omega$.

Lemma 2.2. Let $u \in C^{2}(\bar{\Omega})$ be a weak solution to (1). Then, for any $i=1, \ldots, n$, and any $\varphi \in C^{1}(\bar{\Omega})$, we have

$$
\begin{aligned}
& \int_{\Omega}\left\langle A(\nabla u) \nabla u_{i}, \nabla \varphi\right\rangle d x-\int_{\Omega} f^{\prime}(u) u_{i} \varphi d x \\
= & \int_{\partial \Omega} a(|\nabla u|)\langle\nabla u, \nabla \varphi\rangle \nu_{i} d \sigma-\int_{\partial \Omega} f(u) \varphi \nu_{i} d \sigma+\int_{\partial \Omega} h(u) \varphi_{i} d \sigma .
\end{aligned}
$$

Proof. By Lemma 2.2 in [17] we have that

$$
\text { the map } \quad x \rightarrow W(x):=a(|\nabla u(x)|) \nabla u(x) \quad \text { belongs to } W_{l o c}^{1,1}\left(\Omega, \mathbb{R}^{n}\right),
$$

By Stampacchia's Theorem (see e.g. [32, Theorem 6:19]), we get that $\partial_{i} W=0$ for almost any $x \in\{W=0\}$. In the same way, by Stampacchia's Theorem and (A2), it can be proven that $\nabla u_{i}(x)=0$, and hence $A(\nabla u(x)) \nabla u_{i}(x)=0$, for almost any $x \in\{\nabla u=0\} \cap \Omega$. Moreover,

$$
\partial_{i} W=A(\nabla u) \nabla u_{i} \quad \text { a.e. in } \Omega .
$$


Applying (6) with $\varphi$ replaced by $\varphi_{i}$, making use of (12) and the integration by parts formula, we get

$$
\begin{aligned}
0= & \int_{\Omega} a(|\nabla u|)\left\langle\nabla u, \nabla \varphi_{i}\right\rangle d x+\int_{\partial \Omega} h(u) \varphi_{i} d \sigma-\int_{\Omega} f(u) \varphi_{i} d x \\
= & \int_{\Omega}\left[\partial_{i}(a(|\nabla u|)\langle\nabla u, \nabla \varphi\rangle)-\left\langle A(\nabla u) \nabla u_{i}, \nabla \varphi\right\rangle\right] d x \\
& \quad+\int_{\partial \Omega} h(u) \varphi_{i} d \sigma-\int_{\Omega}\left[\partial_{i}(f(u) \varphi)-f^{\prime}(u) u_{i} \varphi\right] d x \\
= & -\int_{\Omega}\left[\left\langle A(\nabla u) \nabla u_{i}, \nabla \varphi\right\rangle-f^{\prime}(u) u_{i} \varphi\right] d x \\
& \quad+\int_{\partial \Omega} a(|\nabla u|)\langle\nabla u, \nabla \varphi\rangle \nu_{i} d \sigma-\int_{\partial \Omega} f(u) \varphi \nu_{i} d \sigma+\int_{\partial \Omega} h(u) \varphi_{i} d \sigma
\end{aligned}
$$

which proves (11).

Concerning the proof of Lemma 2.2 and the fact that we separate the analysis of the region in which the gradient vanishes with respect to the one in which the gradient differs from zero, we remark that, in the generality considered here, it is possible that both of these sets have positive Lebesgue measure simultaneously. See for instance the examples in Propositions 7.2 and 7.3 in [17] dealing with one-dimensional $p$-Laplace equations with $p>$ 2.

From now on, we use $A$ and $a$, as a short-hand notation for $A(\nabla u)$ and $a(|\nabla u|)$, respectively.

We now provide the proof of Theorem 1.3 .

Proof of Theorem 1.3. We start by observing that by Stampacchia's Theorem we get

$$
\begin{gathered}
\nabla|\nabla u|(x)=0 \quad \text { a.e. } x \in\{|\nabla u|=0\}, \\
\nabla u_{j}(x)=0 \quad \text { a.e. } x \in\{|\nabla u|=0\} \subseteq\left\{u_{j}=0\right\} \quad \text { for any } j=1, \ldots, n .
\end{gathered}
$$

Now, let $\varphi \in C^{1}(\bar{\Omega})$ and $i=1, \ldots, n$. Using (11) with test function $u_{i} \varphi^{2}$, we obtain that

$$
\begin{aligned}
& \int_{\Omega}\left\langle A \nabla u_{i}, \nabla\left(u_{i} \varphi^{2}\right)\right\rangle d x-\int_{\Omega} f^{\prime}(u) u_{i}^{2} \varphi^{2} d x \\
= & \int_{\partial \Omega} a\left\langle\nabla u, \nabla\left(u_{i} \varphi^{2}\right)\right\rangle \nu_{i} d \sigma-\int_{\partial \Omega} f(u) u_{i} \varphi^{2} \nu_{i} d \sigma+\int_{\partial \Omega} h(u) \partial_{i}\left(u_{i} \varphi^{2}\right) d \sigma \\
= & \int_{\partial \Omega} a\left\langle\nabla u, \nabla\left(u_{i} \varphi^{2}\right)\right\rangle \nu_{i} d \sigma-\int_{\partial \Omega} f(u) u_{i} \varphi^{2} \nu_{i} d \sigma+\int_{\partial \Omega} h(u)\left(u_{i i} \varphi^{2}+2 u_{i} \varphi \varphi_{i}\right) d \sigma .
\end{aligned}
$$


Hence, summing over $i=1, \ldots, n$ and recalling (13) and (14), we get

$$
\begin{aligned}
& \int_{\{\nabla u \neq 0\} \cap \Omega}\left(\sum_{i=1}^{n}\left\langle A \nabla u_{i}, \nabla u_{i}\right\rangle \varphi^{2}+2 \varphi|\nabla u|\langle A \nabla \varphi, \nabla|\nabla u|\rangle\right) d x-\int_{\Omega} f^{\prime}(u)|\nabla u|^{2} \varphi^{2} d x \\
= & \int_{\Omega}\left(\sum_{i=1}^{n}\left\langle A \nabla u_{i}, \nabla\left(u_{i} \varphi^{2}\right)\right\rangle-f^{\prime}(u)|\nabla u|^{2} \varphi^{2}\right) d x \\
= & \int_{\partial \Omega} \sum_{i=1}^{n} a\left\langle\nabla u, \nabla\left(u_{i} \varphi^{2}\right)\right\rangle \nu_{i} d \sigma-\int_{\partial \Omega} f(u) \varphi^{2}\langle\nabla u, \nu\rangle d \sigma+\int_{\partial \Omega} h(u)\left(\Delta u \varphi^{2}+\nabla u \cdot \nabla\left(\varphi^{2}\right)\right) d \sigma \\
= & \int_{\partial \Omega} a\left\langle\nabla u, \partial_{\nu} \nabla u\right\rangle \varphi^{2} d \sigma+\int_{\partial \Omega} a\left\langle\nabla u, \nabla\left(\varphi^{2}\right)\right\rangle \partial_{\nu} u d \sigma-\int_{\partial \Omega} f(u) \varphi^{2}\langle\nabla u, \nu\rangle d \sigma \\
& \quad+\int_{\partial \Omega} h(u)\left(\Delta u \varphi^{2}+\left\langle\nabla u, \nabla\left(\varphi^{2}\right)\right\rangle\right) d \sigma .
\end{aligned}
$$

Now we recall the Robin condition in (11) and we obtain that

$$
\begin{aligned}
& \int_{\{\nabla u \neq 0\} \cap \Omega}\left(\sum_{i=1}^{n}\left\langle A \nabla u_{i}, \nabla u_{i}\right\rangle \varphi^{2}+2 \varphi|\nabla u|\langle A \nabla \varphi, \nabla|\nabla u|\rangle\right) d x-\int_{\Omega} f^{\prime}(u)|\nabla u|^{2} \varphi^{2} d x \\
= & \int_{\partial \Omega} a\left\langle\nabla u, \partial_{\nu} \nabla u\right\rangle \varphi^{2} d \sigma-\int_{\partial \Omega} h(u)\left\langle\nabla u, \nabla\left(\varphi^{2}\right)\right\rangle d \sigma-\int_{\partial \Omega} f(u) \partial_{\nu} u \varphi^{2} d \sigma \\
& \quad+\int_{\partial \Omega} h(u)\left(\Delta u \varphi^{2}+\left\langle\nabla u, \nabla\left(\varphi^{2}\right)\right\rangle\right) d \sigma \\
= & \int_{\partial \Omega} a\left\langle\nabla u, \partial_{\nu} \nabla u\right\rangle \varphi^{2} d \sigma-\int_{\partial \Omega} f(u) \partial_{\nu} u \varphi^{2} d \sigma+\int_{\partial \Omega} h(u) \Delta u \varphi^{2} d \sigma .
\end{aligned}
$$

On the other hand, using (77) with test function $|\nabla u| \varphi$ and recalling again (13), we then get

$$
\begin{aligned}
0 \leq & \int_{\Omega}\left(\langle A \nabla(|\nabla u| \varphi), \nabla(|\nabla u| \varphi)\rangle-f^{\prime}(u)|\nabla u|^{2} \varphi^{2}\right) d x+\int_{\partial \Omega} h^{\prime}(u)|\nabla u|^{2} \varphi^{2} d \sigma \\
= & \int_{\Omega}|\nabla u|^{2}\langle A \nabla \varphi, \nabla \varphi\rangle d x \\
& +\int_{\{\nabla u \neq 0\} \cap \Omega}\left(\langle A \nabla|\nabla u|, \nabla|\nabla u|\rangle \varphi^{2}+\langle A \nabla \varphi, \nabla|\nabla u|\rangle 2 \varphi|\nabla u|\right) d x \\
& -\int_{\{\nabla u \neq 0\} \cap \Omega} f^{\prime}(u)|\nabla u|^{2} \varphi^{2} d x+\int_{\partial \Omega} h^{\prime}(u)|\nabla u|^{2} \varphi^{2} d \sigma .
\end{aligned}
$$


Putting together (15) and (16), we conclude that

$$
\begin{aligned}
0 \leq \int_{\Omega}|\nabla u|^{2}\langle A \nabla \varphi, \nabla \varphi\rangle d x \\
\quad+\int_{\{\nabla u \neq 0\} \cap \Omega}\left[\langle A \nabla|\nabla u|, \nabla|\nabla u|\rangle-\sum_{i=1}^{n}\left\langle A \nabla u_{i}, \nabla u_{i}\right\rangle\right] \varphi^{2} d x \\
\quad+\int_{\partial \Omega} a\left\langle\nabla u, \partial_{\nu} \nabla u\right\rangle \varphi^{2} d \sigma-\int_{\partial \Omega} f(u) \partial_{\nu} u \varphi^{2} d \sigma \\
\quad+\int_{\partial \Omega} h(u) \Delta u \varphi^{2} d \sigma+\int_{\partial \Omega} h^{\prime}(u)|\nabla u|^{2} \varphi^{2} d \sigma
\end{aligned}
$$

which is the thesis.

We complete this section by proving Corollary 1.4 .

Proof of Corollary 1.4. By Lemma 2.3 in [17] we see that

$$
\begin{aligned}
& \langle A \nabla|\nabla u|, \nabla|\nabla u|\rangle-\sum_{i=1}^{n}\left\langle A \nabla u_{i}, \nabla u_{i}\right\rangle \\
= & a\left[\left.|\nabla| \nabla u\right|^{2}-\sum_{i=1}^{n}\left|\nabla u_{i}\right|^{2}\right]-a^{\prime}|\nabla u|\left|\nabla_{T}\right| \nabla u \|^{2} .
\end{aligned}
$$

Therefore, using (5) we get

$$
\begin{aligned}
& \langle A \nabla|\nabla u|, \nabla|\nabla u|\rangle-\sum_{i=1}^{n}\left\langle A \nabla u_{i}, \nabla u_{i}\right\rangle \\
& =-\left.\lambda_{1}\left|\nabla_{T}\right| \nabla u\right|^{2}-a(|\nabla u|)\left(\sum_{i=1}^{n}\left|\nabla u_{i}\right|^{2}-\left.\left|\nabla_{T}\right| \nabla u\right|^{2}-\left.|\nabla| \nabla u\right|^{2}\right) .
\end{aligned}
$$

Notice that the quantity

$$
\sum_{i=1}^{n}\left|\nabla u_{i}\right|^{2}-\left.\left|\nabla_{T}\right| \nabla u\right|^{2}-\left.|\nabla| \nabla u\right|^{2}
$$

has a geometric interpretation, in the sense that it can be expressed in terms of the principal curvatures of level sets of $u$. Indeed, the following formula holds (see [17, 40, 41])

$$
\sum_{i=1}^{n}\left|\nabla u_{i}\right|^{2}-\left.|\nabla| \nabla u\right|^{2}-\left|\nabla_{T}\right| \nabla u||^{2}=|\nabla u|^{2} \sum_{j=1}^{n-1} k_{j}^{2} \quad \text { on } L_{u, x} \cap\{\nabla u \neq 0\} .
$$


With this, formula (8) becomes

$$
\begin{aligned}
& \int_{\Omega}\left[\lambda_{1}\left|\nabla_{T}\right| \nabla u||^{2}+a|\nabla u|^{2} \sum_{j=1}^{n-1} k_{j}^{2}\right] \varphi^{2} d x \\
& \quad+\int_{\partial \Omega}\left(f(u) \partial_{\nu} u-a\left\langle\nabla u, \partial_{\nu} \nabla u\right\rangle-h(u) \Delta u-h^{\prime}(u)|\nabla u|^{2}\right) \varphi^{2} d \sigma \\
& \leq \int_{\Omega}|\nabla u|^{2}\langle A \nabla \varphi, \nabla \varphi\rangle d x
\end{aligned}
$$

which is the desired inequality.

\section{Proof of Theorem 1.5}

In this section we will use formula (9) to prove Theorem 1.5, following the approach introduced in [14] and then developed in [17, 12].

We start with the following result:

Lemma 3.1. Let $\Omega \subset \mathbb{R}^{n}$ be an open convex set with boundary of class $C^{2}$ and let $a \in$ $C_{l o c}^{1,1}((0,+\infty))$ satisfying (2). Let $u \in C^{2}(\bar{\Omega})$, with $\partial_{\nu} u=0$ on $\partial \Omega$.

Then, at each point $x \in \partial \Omega$ it holds

$$
a(|\nabla u(x)|)\left\langle\nabla u(x), \partial_{\nu} \nabla u(x)\right\rangle \leq 0 .
$$

Proof. If $\nabla u(x) \neq 0$ then the thesis follows as in [4, Theorem 2] (see also [12, Lemma 2.1]). If $\nabla u(x)=0$ then $\left\langle\nabla u(x), \partial_{\nu} \nabla u(x)\right\rangle=0$ and the thesis follows as well.

We also need the following result, whose proof is similar to that of Corollary 2.6 in [12] and so is omitted.

Lemma 3.2. Let $x_{o} \in \Omega$, with $\nabla u\left(x_{o}\right) \neq 0$. Suppose that

$$
\langle A \nabla|\nabla u|, \nabla|\nabla u|\rangle-\sum_{i=1}^{n}\left\langle A \nabla u_{i}, \nabla u_{i}\right\rangle=0 .
$$

Then, each connected component of the level sets of $u$ must be an $(n-2)$-dimensional hyperplane intersected $\Omega$.

With these two results, we can now complete the proof of Theorem 1.5.

Proof of Theorem 1.5. The proof is based on the choice of the test function in (9). Indeed, we let $\varphi \equiv 1$ in (9), we recall the Neumann boundary condition in (1) and we obtain that

$$
\begin{gathered}
\int_{\Omega}\left[\lambda_{1}\left|\nabla_{T}\right| \nabla u||^{2}+a|\nabla u|^{2} \sum_{j=1}^{n-1} k_{j}^{2}\right] \varphi^{2} d x-\int_{\partial \Omega} a\left\langle\nabla u, \partial_{\nu} \nabla u\right\rangle \varphi^{2} d \sigma \\
\leq \int_{\Omega}|\nabla u|^{2}\langle A \nabla \varphi, \nabla \varphi\rangle d x=0 .
\end{gathered}
$$

Then, recalling Lemma 3.1, we conclude that

$$
k_{j}(x)=0, \quad\left|\nabla_{T}\right| \nabla u||(x)=0
$$


for every $j=1, \ldots, n-1$ and every $x \in\{\nabla u \neq 0\}$, and

$$
\left\langle\nabla u, \partial_{\nu} \nabla u\right\rangle=0 \quad \text { on } \partial \Omega .
$$

Having proved (23) and (24), the thesis follows as in the proof of Theorem 1.2 in [12]. We give the details here for the sake of completeness.

We first claim that

$$
u \text { is constant along } \partial \Omega \text {. }
$$

To check this, we argue towards a contradiction, supposing that there exist $x, y \in \partial \Omega$ such that $u(x) \neq u(y)$. From Lemma 2.2 in [12, we know that we can connect $x$ and $y$ with a continuous path $\sigma:[0,1] \rightarrow \partial \Omega$. So, we define $\zeta(t):=u(\sigma(t))$, and we see that $\zeta(0) \neq \zeta(1)$. Hence, we can find $\bar{t} \in(0,1)$ such that $\dot{\zeta}(\bar{t}) \neq 0$, namely

$$
0 \neq \dot{\zeta}(\bar{t})=\nabla u(\sigma(\bar{t})) \cdot \dot{\sigma}(\bar{t})
$$

We also let $\bar{x}:=\sigma(\bar{t})$. Up to a change of coordinates, we may suppose that the exterior normal of $\partial \Omega$ at $\bar{x}$ coincides with $-e_{n}$, and therefore $\Omega$ can be written in normal coordinates as the epigraph of a function $\gamma \in C^{2}\left(\mathbb{R}^{n-1}\right)$ near $\bar{x}$.

Since, by assumptions, the principal curvatures of $\partial \Omega$ are positive, we have that the Hessian of $\gamma$ is positive definite.

On the other hand, by (24) here and formula (2.1) in [12, we have that

$$
0=-\left\langle\nabla u(\bar{x}), \partial_{\nu} \nabla u(\bar{x})\right\rangle=\sum_{i, j=1}^{n-1} \gamma_{i j}\left(\bar{x}^{\prime}\right) u_{i}(\bar{x}) u_{j}(\bar{x}) .
$$

This and (27) imply that $u_{i}(\bar{x})=0$ for any $i=1, \ldots, n-1$.

Furthermore, we have that $u_{n}(\bar{x})=-\partial_{\nu} u(\bar{x})=0$, thanks to the choice of the coordinate system and the Neumann condition. This and (28) give that $\nabla u(\bar{x})=0$, in contradiction with (26), which proves (25).

Now, we let $c$ be the value attained by $u$ along $\partial \Omega$, as given by (25), and we complete the proof of Theorem 1.5, by showing that

$u$ is constant in $\Omega$.

Indeed, if this was not true, then we would have that

$$
\{x \in \Omega \text { s.t. } \nabla u(x) \neq 0\} \neq \varnothing .
$$

As a consequence, we can take an arbitrary point $x_{0} \in \Omega$ such that $\nabla u\left(x_{0}\right) \neq 0$. We also let $L\left(x_{0}\right)$ be the connected component of the level set of $u$ in $\Omega$ passing through $x_{0}$.

Furthermore, we notice that assumption (21) in Lemma 3.2 is satisfied, thanks to the geometric observation in formulas (19) and (23). Therefore, we can use Lemma 3.2 to say that

$$
L\left(x_{0}\right)=\left\{\omega \cdot\left(x-x_{0}\right)=0\right\} \cap \Omega,
$$

for a suitable $\omega \in S^{n-1}$, possibly depending on $x_{0}$.

Now we take a vector $\varpi$ orthogonal to $\omega$ (of course, $\varpi$ may also depend on $x_{0}$ ), and we consider the straight line

$$
\left\{x_{0}+\varpi t, t \in \mathbb{R}\right\} .
$$


Since the domain $\Omega$ is bounded, such a line must intersect $\partial \Omega$, that is there exists $t_{0}$ such that $x_{0}+\varpi t_{0} \in \partial \Omega$. Accordingly, by (25),

$$
u\left(x_{0}+\varpi t_{0}\right)=c .
$$

On the other hand, (31) implies that

$$
u\left(x_{0}+\varpi t_{0}\right)=u\left(x_{0}\right) .
$$

This and (32) give that $u\left(x_{0}\right)=c$.

Notice that this holds for any point $x_{0} \in \Omega$ such that $\nabla u\left(x_{0}\right) \neq 0$. Therefore, we have established that

$$
u(x)=c \text { for any } x \in \Omega \cap\{\nabla u \neq 0\} .
$$

Since the above identity also holds on $\partial \Omega$ and since $u$ is constant in each component of $\Omega \cap\{\nabla u=0\}$, we obtain that $u(x)=c$ for any $x \in \Omega$, and so $\nabla u$ vanishes identically in $\Omega$.

This gives a contradiction with (30), thus proving (29). Hence, the proof of Theorem 1.5 is completed.

\section{Proof of Theorem 1.6}

We argue towards a contradiction, by supposing that $u$ is stable. So, we can use Corollary 1.4, in particular, we choose $\varphi \equiv 1$ in (9), and we obtain that

$$
\begin{aligned}
\int_{\Omega}\left[\left|\nabla_{T}\right| \nabla u||^{2}+|\nabla u|^{2} k_{1}^{2}\right] d x & \\
& +\int_{\partial \Omega}\left(f(u) \partial_{\nu} u-\left\langle\nabla u, \partial_{\nu} \nabla u\right\rangle-\alpha u \Delta u-\alpha|\nabla u|^{2}\right) d \sigma \\
\leq & \int_{\Omega}|\nabla u|^{2}\langle A \nabla \varphi, \nabla \varphi\rangle d x=0 .
\end{aligned}
$$

Thus, recalling the Robin boundary condition

$$
\partial_{\nu} u+\alpha u=0 \quad \text { on } \partial \Omega,
$$

we get

$$
\begin{aligned}
\int_{\Omega}[ & \left.\left.\left|\nabla_{T}\right| \nabla u\right|^{2}+|\nabla u|^{2} k_{1}^{2}\right] d x \\
& -\int_{\partial \Omega}\left(\alpha f(u) u+\left\langle\nabla u, \partial_{\nu} \nabla u\right\rangle+\alpha u \Delta u+\alpha|\nabla u|^{2}\right) d \sigma \leq 0 .
\end{aligned}
$$

Moreover, since $u \in C^{2}(\bar{\Omega})$, the equation

$$
\Delta u+f(u)=0
$$

holds up to the boundary of $\Omega$, and so (34) becomes

$$
\int_{\Omega}\left[\left.\left|\nabla_{T}\right| \nabla u\right|^{2}+|\nabla u|^{2} k_{1}^{2}\right] d x-\int_{\partial \Omega}\left(\left\langle\nabla u, \partial_{\nu} \nabla u\right\rangle+\alpha|\nabla u|^{2}\right) d \sigma \leq 0 .
$$

Also, we observe that

$$
\int_{\Omega}\left[\left.\left|\nabla_{T}\right| \nabla u\right|^{2}+|\nabla u|^{2} k_{1}^{2}\right] d x \geq 0
$$


This and (35) give that

$$
\int_{\partial \Omega}\left(\left\langle\nabla u, \partial_{\nu} \nabla u\right\rangle+\alpha|\nabla u|^{2}\right) d \sigma \geq 0
$$

Now, we suppose that the boundary $\partial \Omega$ is represented by the curve $s \mapsto x(s):=$ $\left(x_{1}(s), x_{2}(s)\right)$, with $s \in[0, \ell]$, being $s$ the arc-length. Therefore, in a neighborhood of the boundary, a point $x \in \Omega$ can be written as

$$
x(s, t)=x(s)-t \nu(s),
$$

where $(s, t)$ are the so-called normal coordinates.

In this setting, we recall formulas (2.2) and (2.5) in [2]:

$$
\begin{aligned}
|\nabla u|^{2} & =u_{s}^{2}+u_{t}^{2} \\
\text { and }\left\langle\nabla u, \partial_{\nu} \nabla u\right\rangle & =-(\alpha+\kappa) u_{s}^{2}-\kappa \alpha^{2} u^{2}+\alpha u u_{s s}+\alpha f(u) u,
\end{aligned}
$$

being $\kappa$ the curvature of $\partial \Omega$. Here, $u_{s}, u_{t}, u_{s s}$ and $u_{t t}$ denote the first and the second derivatives with respect to the normal coordinates.

Also, the boundary condition in (33) reads as

$$
u_{t}=\alpha u
$$

Finally, the following formula holds:

$$
u_{t t}-\kappa u_{t}+u_{s s}+f(u)=0 \quad \text { on } \partial \Omega,
$$

see e.g. the formula above (2.5) in [2].

These observations imply that

$$
\begin{aligned}
& \left\langle\nabla u, \partial_{\nu} \nabla u\right\rangle+\alpha|\nabla u|^{2} \\
= & -(\alpha+\kappa) u_{s}^{2}-\kappa \alpha^{2} u^{2}+\alpha u u_{s s}+\alpha f(u) u+\alpha\left(u_{s}^{2}+u_{t}^{2}\right) \\
= & -\kappa u_{s}^{2}-\kappa \alpha^{2} u^{2}+\alpha u u_{s s}+\alpha f(u) u+\alpha u_{t}^{2} \\
= & -\kappa u_{s}^{2}-\kappa \alpha^{2} u^{2}+\alpha u u_{s s}+\alpha f(u) u+\alpha^{3} u^{2} .
\end{aligned}
$$

Hence, integrating over $\partial \Omega$,

$$
\begin{aligned}
& \int_{\partial \Omega}\left(\left\langle\nabla u, \partial_{\nu} \nabla u\right\rangle+\alpha|\nabla u|^{2}\right) d s \\
= & \int_{\partial \Omega}\left(-\kappa u_{s}^{2}-\kappa \alpha^{2} u^{2}+\alpha u u_{s s}+\alpha f(u) u+\alpha^{3} u^{2}\right) d s .
\end{aligned}
$$

Now, we observe that

$$
\int_{\partial \Omega} u u_{s s} d s=-\int_{\partial \Omega} u_{s}^{2} d s
$$


and so, using this into (37), we conclude that

$$
\begin{aligned}
& \int_{\partial \Omega}\left(\left\langle\nabla u, \partial_{\nu} \nabla u\right\rangle+\alpha|\nabla u|^{2}\right) d s \\
= & \int_{\partial \Omega}\left(-\kappa u_{s}^{2}-\kappa \alpha^{2} u^{2}-\alpha u_{s}^{2}+\alpha f(u) u+\alpha^{3} u^{2}\right) d s \\
= & \int_{\partial \Omega}\left[\alpha^{2} u^{2}\left(\frac{f(u)}{\alpha u}-\kappa+\alpha\right)-(\alpha+\kappa) u_{s}^{2}\right] d s \\
< & 0,
\end{aligned}
$$

thanks to (10). This is in contradiction with (36), and so the proof of Theorem [1.6 is complete.

\section{REFERENCES}

[1] Arrieta, J. M., Carvalho, A. N.: Spectral convergence and nonlinear dynamics of reaction-diffusion equations under perturbations of the domain, J. Differential Equations 199, no. 1, 143-178 (2004).

[2] Bandle, C., Mastrolia, P., Monticelli, D. D., Punzo, F.: On the stability of solutions of semilinear elliptic equations with Robin boundary conditions on Riemannian manifolds, SIAM J. Math. Anal. 48, no. 1, 122-151 (2016).

[3] Bolikowski, Ł., Gokieli, M., Varchon, N.: The Neumann problem in an irregular domain, Interfaces Free Bound. 12, no. 4, 443-462 (2010).

[4] Casten, R. G., Holland, C. J.: Instability results for reaction diffusion equations with Neumann boundary conditions, J. Differential Equations 27, no. 2, 266-273 (1978).

[5] Cesaroni, A., Novaga, M., Pinamonti, A.: One-dimensional symmetry for semilinear equations with unbounded drift, Commun. Pure Appl. Anal. 12, no. 5, 2203-2211 (2013).

[6] Cesaroni, A., Novaga, M., Valdinoci, E.: A simmetry result for the Ornstein-Uhlenbeck operator, Discrete Contin. Dyn. Syst. 34, no. 6, 2451-2467 (2014).

[7] De Oliveira, L. A. F., Pereira, A. L., Pereira, M. C.: Continuity of attractors for a reaction-diffusion problem with respect to variations of the domain, Electron. J. Differential Equations 100, 18 pp. (2005).

[8] Dipierro, S.: Geometric inequalities and symmetry results for elliptic systems, Discrete Contin. Dyn. Syst. 33, no. 8, 3473-3496 (2013).

[9] Dipierro, S., Pinamonti, A.: A geometric inequality and a symmetry result for elliptic systems involving the fractional Laplacian, J. Differential Equations 255, no. 1, 85-119. (2013).

[10] Dipierro, S., Pinamonti, A.: Symmetry results for stable and monotone solutions to fibered systems of PDEs, Commun. Contemp. Math. 17, no. 4, 1450035, 22 pp. (2015).

[11] Dipierro, S., Pinamonti, A., Valdinoci, E.: Classification of stable solutions for boundary value problems with nonlinear boundary conditions on Riemannian manifolds with nonnegative Ricci curvature. To appear in Adv. Nonlinear Anal. arXiv:1710.07329

[12] Dipierro, S., Soave, N., Valdinoci, E.: On stable solutions of boundary reaction-diffusion equations and applications to nonlocal problems with Neumann data, Indiana Univ. Math. J. 67, no. 1, 429-469 (2018).

[13] Dupaigne, L.: Stable solutions of elliptic partial differential equations, vol. 143 of Chapman \& Hall/CRC Monographs and Surveys in Pure and Applied Mathematics, CH/CRC, Boca Raton, FL (2011).

[14] Farina, A.: Propriétés qualitatives de solutions d'équations et systèmes d'équations non-linéaires, Habilitation à diriger des recherches, Paris VI, (2002).

[15] Farina, A., Mari, L., Valdinoci, E.: Splitting theorems, symmetry results and overdetermined problems for Riemannian manifolds, Comm. Partial Differential Equations 38, no. 10, 1818-1862 (2013).

[16] Farina, A., Novaga, M., Pinamonti, A.: Symmetry results for nonlinear elliptic operators with unbounded drift, NoDEA Nonlinear Differential Equations Appl. 21, no. 6, 869-883 (2014). 
[17] Farina, A., Sciunzi, B., Valdinoci, E.: Bernstein and De Giorgi type problems: new results via a geometric approach, Ann. Sc. Norm. Super. Pisa Cl. Sci. (5) 7, no. 4, 741-791 (2008).

[18] Farina, A., Sciunzi, B., Valdinoci, E.: On a Poincaré type formula for solutions of singular and degenerate elliptic equations, Manuscripta Math. 132, no. 3-4, 335-342 (2010).

[19] Farina, A., Sire, Y., Valdinoci, E.: Stable solutions of elliptic equations on Riemannian manifolds with Euclidean coverings, Proc. Amer. Math. Soc. 140, no. 3, 927-930 (2012).

[20] Farina, A., Sire, Y., Valdinoci, E.: Stable solutions of elliptic equations on Riemannian manifolds, J. Geom. Anal. 23, no. 3, 1158-1172 (2013).

[21] Farina, A., Valdinoci, E.: The state of the art for a conjecture of De Giorgi and related problems. In: Du, Y., Ishii, H., Lin, W.-Y. (eds.), Recent Progress on Reaction Diffusion System and Viscosity Solutions. Series on Advances in Mathematics for Applied Sciences, 372 World Scientific, Singapore (2008).

[22] Fazly, M., Ghoussoub, N.: De Giorgi type results for elliptic systems, Calc. Var. Partial Differential Equations 47, no. 3-4, 809-823 (2013).

[23] Hale, J. K., Vegas, J.: A nonlinear parabolic equation with varying domain, Arch. Rational Mech. Anal. 86, no. 2, 99-123 (1984).

[24] Ferrari, F., Pinamonti, A.: Nonexistence results for semilinear equations in Carnot groups, Anal. Geom. Metr. Spaces 1, 130-146 (2013).

[25] Ferrari, F., Valdinoci, E.: A geometric inequality in the Heisenberg group and its applications to stable solutions of semilinear problems, Math. Ann. 343, no. 2, 351-370 (2009).

[26] Giusti, E.: On the equation of surfaces of prescribed mean curvature. Existence and uniqueness without boundary conditions, Invent. Math. 46, no. 2, 111-137 (1978).

[27] Jimbo, S.: On a semilinear diffusion equation on a Riemannian manifold and its stable equilibrium solutions. Proc. Japan Acad. Ser. A Math. Sci. 60, no. 10, 349-352 (1984).

[28] Jimbo, S.: The singularly perturbed domain and the characterization for the eigenfunctions with Neumann boundary condition, J. Differential Equations 77, no. 2, 322-350 (1989).

[29] Jimbo, S.: Singular perturbation of domains and semilinear elliptic equations. III, Hokkaido Math. J. 33, no. 1, 11-45 (2004).

[30] Jimbo, S., Morita, Y.: Remarks on the behavior of certain eigenvalues on a singularly perturbed domain with several thin channels, Comm. Partial Differential Equations 17, no. 3-4, 523-552 (1992).

[31] Ladyzhenskaya, O., Uraltseva, N.: Linear and Quasilinear Elliptic Equations, Academic Press, New York, (1968).

[32] Lieb, H. H., Loss, M.: Analysis, vol. 14 of Graduate Studies in Mathematics, AMS, Providence, RI (1997).

[33] Lieberman, G.: Boundary regularity for solutions of degenerate elliptic equations, Nonlinear Anal. 12, no. 11, 1203-1219 (1988).

[34] Ma, X.-N., Wang, P.-H., Wei, W.: Constant mean curvature surfaces and mean curvature flow with non-zero Neumann boundary conditions on strictly convex domains, J. Funct. Anal. 274, no. 1, 252-277 (2018).

[35] Matano, H.: Asymptotic behavior and stability of solutions of semilinear diffusion equations, Publ. Res. Inst. Math. Sci. 15, no. 2, 401-454 (1979).

[36] Morita, Y., Jimbo, S.: Ordinary differential equations (ODEs) on inertial manifolds for reactiondiffusion systems in a singularly perturbed domain with several thin channels, J. Dynam. Differential Equations 4, no. 1, 65-93 (1992).

[37] Pinamonti, A., Valdinoci, E.: A geometric inequality for stable solutions of semilinear elliptic problems in the Engel group, Ann. Acad. Sci. Fenn. Math. 37, no. 2, 357-373 (2012).

[38] Sire, Y., Valdinoci, E.: Fractional Laplacian phase transitions and boundary reactions: a geometric inequality and a symmetry result. J. Funct. Anal. 256, no. 6, 1842-1864 (2009).

[39] Sire, Y., Valdinoci, E.: Rigidity results for some boundary quasilinear phase transitions. Comm. Partial Differential Equations 34, no. 7-9, 765-784 (2009).

[40] Sternberg, P., Zumbrun, K.: A Poincaré inequality with applications to volume-constrained areaminimizing surfaces, J. Reine Angew. Math. 503, 63-85 (1998). 
[41] Sternberg, P., Zumbrun, K.: Connectivity of phase boundaries in strictly convex domains, Arch. Ration. Mech. Anal. 141, no. 4, 375-400 (1998).

[42] Vegas, J. M.: Bifurcations caused by perturbing the domain in an elliptic equation, J. Differential Equations 48, no. 2, 189-226 (1983).

Dipartimento di Matematica "Federigo Enriques", Università degli studi di Milano, Via SAldini 50, 20133 Milano (Italy).

E-mail address: serena.dipierro@unimi.it

Dipartimento di Matematica, Università di Trento, Via Sommarive 14, 38050 Povo, Trento (ITALY).

E-mail address: Andrea.Pinamonti@gmail.com

Dipartimento di Matematica "Federigo Enriques", Università degli studi di Milano, Via Saldini 50, 20133 Milano (Italy), and School of Mathematics and Statistics, University of Melbourne, Grattan Street, Parkville, ViC-3010 Melbourne (Australia), and Istituto di Matematica Applicata e Tecnologie Informatiche, Via Ferrata 1, 27100 Pavia (Italy).

E-mail address: enrico.valdinoci@unimi.it 